\title{
RESOLUCIÓN DE CONFLICTOS EN LA ESCUELA: UNA HERRAMIENTA PARA LA CULTURA DE PAZ Y LA CONVIVENCIA
}

\author{
Silvina Funes Lapponi \\ Universidad de Salamanca
}

\begin{abstract}
RESUMEN. La creación de un clima de convivencia pacífica en los centros educativos, por medio de fomentar habilidades sociales específicas, como la mejora de la comunicación, la negociación y la mediación, es una forma eficaz de hacer efectiva la educación para la paz en el día a día y no sólo por la simple enunciación de datos o declaraciones de principios que a menudo son vistos por los alumnos como una parte más del currículum.

Una cultura de paz en lo cotidiano concibe al conflicto como oportunidades de crecimiento personal y para los grupos, y no como meros problemas de disciplina que deben ser resueltos a cualquier coste y sin preocuparse por la continuidad de la relación.

Desde esta perspectiva, la educación para la paz es una paz en el entorno cotidiano, de resolución de conflictos a pequeña escala, es decir de paz activa, de soluciones y aportaciones constructivas, de protagonismo de los participantes.

ABSTRACT. The creation of a peaceful environment in living together at the educational centers through the promotion of specific social abilities, like better communication, negotiation and mediation, is an effective way to make an efficient peace education by the daily living together, and not only for a simple enunciation of facts or statements of principles, which usually are considered by students like a part of their curriculum.

In peace culture related with our daily coexistence, conflict has to be seen as an opportunity of personal and group growing up, and not like simple problems of discipline that have to be solved one way or another.

Considering this perspective, peace education means a peace in our daily living together, in resolving small degree conflicts, or in other way it means, an active peace, with solutions and constructive contributions as the main rol of the participants.
\end{abstract}

\section{Convivencia y disciplina: el camino de la negociación}

\subsection{Reforma educativa y sociedad actual. Las nuevas necesidades de socialización}

Participación democrática, educación comprensiva, currículum oculto, educación en valores, son principios con los que cada día estamos más familiarizados pero que siguen planteándonos nuevos retos. Su aplicación en la vida cotidiana del centro nos obliga a repensar sobre sus límites, sobre cómo aplicarlos exactamente. ¿Entendemos 
todos lo mismo por participación, libertad, tolerancia, respeto? Lo cierto es que no, y que para la puesta en marcha de iniciativas vinculadas a esta línea de actuación en cada centro, para que en cada comunidad educativa se abra el debate, para que producto de esa discusión surjan los auténticos proyectos educativos de centro, que se ajusten los Reglamentos de régimen interno a las necesidades de cada comunidad educativa, hace falta reflexionar y definir cada una de estas cuestiones.

La delegación cada vez mayor que hacen las familias de la educación de sus hijos a los centros, la influencia de los MMC y el mayor peso de los grupos de iguales en la vida cotidiana de los jóvenes nos sitúa ante nuevos escenarios de socialización que compiten con los de la enseñanza reglada y en los que esta educación tiene la misión de poner un cierto orden.

Muchas veces los jóvenes presuponen, conocen y defienden el ejercicio de sus libertades y derechos, pero no siempre se le dan pautas claras de los deberes y obligaciones que dichas libertades y derechos conllevan. La labor que queda para la educación en estas condiciones es de tal manera excesiva que está desgastando al profesorado en su quehacer cotidiano, sobre-exigido en su trabajo de poner límites a alumnos, que en muchos casos no los tienen, pero que, por otro lado reaccionan y no permiten los que perciben como "abusos de autoridad".

\subsection{Ideas sobre el conflicto}

Intentaremos a continuación profundizar en cuál es la naturaleza del conflicto y su particular problemática en el ámbito escolar, en primer lugar porque naturalmente se lo opone al concepto de paz, para lo que intentaremos demostrar que esto no es así, y en segundo lugar, porque para poder resolverlos, lo primero que deberemos es tener un conocimiento muy claro del mismo, por lo que, para una buena intervención será fundamental una diagnóstico preciso y sin juicio de valor.

Conflicto es toda actividad en la que unos hombres contienden con otros por la consecución de un/os objetivo/s. Implica desarmonía, incompatibilidad, pugna entre dos partes interdependientes. Es un proceso relacional en el que se producen interacciones antagónicas. Puede originarse simplemente en la percepción de divergencia de necesidades o intereses, que no se satisfacen simultáneamente o en forma conjunta, debido a incompatibilidades o diferencias en los valores o en la definición de la situación, también por competencia o por escasez de recursos. Lo que significa que uno va con un objetivo que el otro, probablemente, está dispuesto a obstaculizar o a no facilitar. Jares añade que las personas o grupos en disputa están determinadas por la incompatibilidad, ya sea personal, o en los valores $o$ intereses que sostienen. ${ }^{1} A$ veces, incluso con la intención de infligir daño. (Moore, Ch., 1994, p. 4).

Existe una tendencia a ver el conflicto como algo negativo, como una circunstancia a la que hay que evitar, que se basa en varias razones. En primer lugar, porque se lo asocia automáticamente a la manera en que se suelen resolver, es decir, mediante la violencia, la eliminación o anulación de una de las partes. Además, existe la idea

1. Jares, X., El lugar del conflicto en la organización escolar, en Revista Iberoamericana de Educación, № 15, pág. 54. 
muy extendida de que hacer frente al conflicto implica invertir mucho tiempo -probablemente ya de por sí escaso en el ámbito educativo- y que, al mismo tiempo, se va a pasar un momento de una gran tensión, e incluso sufrimiento. En estos casos, la forma de enfrentar el conflicto suele ser su evitación.

Existen en el sistema educativo una serie de prejuicios asociados al conflicto; no se percibe el aprendizaje que puede proporcionar el cometer un error, un fracaso. Rotula y condena al acusado. En consecuencia, el sistema educativo no es una institución que facilite y motive el aprendizaje y el crecimiento a partir de los errores, sino la vergüenza y el temor a cometerlos. El conflicto debe ocultarse, negarse o disimularse. El conflicto es obsceno, avergüenza, pone en evidencia nuestra incompetencia. Un modelo de educación entendido así, no respeta la auténtica diversidad de las personas, sino que premia y estimula al que acepta la homogeneización; en eso consiste el ser aprobado por el sistema, en su capacidad de adaptación a su lenguaje, a sus normas, a su modus operandi. Esto no ayuda a fomentar ni en los docentes ni en sus alumnos la construcción de un modelo adecuado de cultura de paz.

El conflicto está culturalmente cargado de una connotación negativa, pero el tipo de vínculo que posea el grupo, sus motivaciones y su comunicación pueden darle a la resolución del conflicto una dimensión positiva, ya que es parte integral del sistema, es decir, no es exclusivo de grupos mal cohesionados o con vínculos patológicos, sino que es intrínseco a la vida de los grupos, sólo que será destructivo o constructivo dependiendo del tipo de relación y comunicación que posea cada grupo y de las actitudes que asuman hacia el conflicto. El conflicto no es malo en sí mismo.

El conflicto es un proceso, es decir, es construido por las partes en disputa y tiene un surgimiento, un desarrollo y un desenlace, y va trazando un "canal", crea una pauta de interacción. Por eso es tan importante enseñar pautas de resolución cooperativa del conflicto.

Hay una perspectiva del conflicto que nos parece singularmente valiosa, que es la perspectiva crítica. En esta visión, el conflicto es necesario para la transformación de las estructuras, las prácticas y los valores educativos, promoviendo la resolución pacífica y constructiva de conflictos, rescatando en éste, por lo tanto, una dimensión educativa y considerando a las perspectivas celosas del poder como perpetuadoras del status quo. Se le reconoce a esta perspectiva no sólo beneficios al nivel del clima organizativo, sino que también de la gestión, ya que promueve la comunicación, la interdependencia y la autonomía. Esta forma de afrontamiento del conflicto afecta a la micropolítica del centro, por la práctica democrática de toma de decisiones, por la participación y la gestión colaborativa, rechazando la visión instrumental de la enseñanza definida por los resultados obtenidos en función de los objetivos.

Si existe una concepción crítica del conflicto que se transmite en el proceso de enseñanza-aprendizaje, habrá una aproximación a la educación para la paz más cercana a la realidad, al entorno cotidiano.

Por el contrario, una mala regulación del conflicto abrirá paso a generar violencia, con la aparición del deseo de causar el máximo daño posible a la otra parte, entendida desde esta perspectiva como el enemigo, generándose de este modo equilibrios inestables, ya que se incrementa la espiral de violencia. En una situación como la descrita, una relación que se asiente sobre un estilo interaccional de estas características, 
estará impregnada de rechazo hacia el otro, provocando un gran deterioro en la relación. Una regulación positiva de un conflicto también se preocupa por la continuidad de la relación, por el otro, y es más probable la aparición de soluciones estables si es que ésta se ha alcanzado por vía de la cooperación.

El desarrollo de las habilidades negociadoras desde un marco colaborador obliga a tener en cuenta que las necesidades de todos son importantes, y que busquen la forma de satisfacerlas. Por lo tanto, estaremos haciendo a las personas también más solidarias y tolerantes, porque el respeto por el otro no es un valor retórico: no se trata de un otro intangible, sino de un otro distinto, un otro concreto, con sus necesidades y sentimientos.

El desarrollo de este tipo de habilidades también favorece la autonomía moral de las personas.

Todas estas habilidades nos remiten al desarrollo de competencias emocionales, que por medio de cuestiones concretas y de forma práctica ponen en marcha en las personas funciones intelectuales sin dejar de lado los aspectos emocionales, favoreciendo así un crecimiento más integral y un mejor ajuste a su entorno. Estas habilidades deben aprenderse y aplicarse en la vida cotidiana, pero dada la función socializadora atribuida al sistema educativo, lo convierte en un espacio fundamental para el aprendizaje de la convivencia en la sociedad, y por eso mismo el idóneo.

Si la violencia se aprende, también se aprende la no-violencia, y la mejor manera de hacerlo será aprendiendo a prevenirla de forma muy concreta, por medio del aprendizaje de estos métodos, ya que se basan en el diálogo. Podemos proporcionar una herramienta más completa y más armonizadora para la resolución de disputas que la imposición o la ley del más fuerte. No sólo estamos previniendo la violencia, sino también socializando en la no-violencia, lo que transforma a nuestro entorno en un lugar donde se convive mejor.

\subsection{Necesidad de atender a problemas de convivencia. El vacío institucional}

Hoy por hoy, la palabra disciplina goza de mala prensa, y llevados por la moda del lenguaje "políticamente correcto" hablamos de convivencia, influenciados por la -a veces- hipocresía del lenguaje. Este auto-engaño no nos ayuda a llevar a cabo ni un buen programa de convivencia en el centro, ni a poder mantener una disciplina mínima, necesaria tanto para el trabajo cotidiano de clase como para el mantenimiento de un programa de convivencia.

Castigos, amonestaciones, partes, expedientes llenan de papeles el "vacío institucional" por el que las conductas reprobables se clasifican y se traducen en un régimen de sanciones y muchas veces este marco que rige la aplicación de esta documentación se pasa de un centro al otro como si de algo estandarizado se tratara.

La mayoría de los esfuerzos en los centros están orientados a elaborar una tipificación de faltas con sus consecuentes castigos, que acaban siendo una "burocratización" de la disciplina, con un perfil marcadamente punitivo o "ejemplarizante" -como le suelen llamar- que en la mayor parte de los casos es muy poco educativo y mucho menos correctivo, por lo menos para el "castigado". 


\subsection{La respuesta tradicional: la atención como un problema de disciplina}

Cuántas veces nos ha pasado tener a un alumno agresivo, respondón, o simplemente inquieto: el típico alumno disruptivo, da igual el castigo que se le ponga, que se hable con la familia, que intervengan otros profesionales, que no se halla una solución. Iniciamos así el camino de la "escalada de castigos": se agudizan, se hacen cada vez más largos, más frecuentes y no hemos visto ningún resultado.

Es muy común oír al profesorado decir: "no nos temen", "no nos respetan". La cuestión es: cuando las soluciones de siempre no dan respuesta a nuestras necesidades, es que ya no funcionan, y hay que cambiarlas.

En el presente artículo no vamos a dar "LA" solución; simplemente vamos a dar hacer una propuesta. Una que, según qué casos, dará más o menos resultado, pero que no estará demás incorporarla a nuestro repertorio de respuestas, porque en definitiva, lo que importa es hacer más eficiente nuestra capacidad de resolver problemas ante una comunidad educativa cuyas demandas y necesidades son cambiantes y, por lo tanto, deberemos cambiar también nuestra capacidad de reacción ante las mismas, diversificando nuestra posibles respuestas, ajustándolas de acuerdo a los distintos problemas que haya que resolver.

La diferencia fundamental -y por la que no podemos equiparar la palabra convivencia a la de disciplina- es que la convivencia requiere de participación, diálogo, negociación, compromiso, y ése es el cambio sustancial que supone, y no un simple maquillaje de palabras. Supone que para la aplicación de un "castigo" debe haber una "toma de conciencia" de su responsabilidad por la persona que ha "dañado" a la comunidad educativa y su implicación en la reparación del daño.

La palabra disciplina tampoco es lo mismo; en tanto y en cuanto habla más de unas formas y normas de procedimiento que tiene una institución, y la convivencia hace alusión a cómo una comunidad define su "estar juntos", de eso se trata, de "VIVIR CON", suponiendo una definición de las relaciones institucionales más horizontales y dialogantes y menos verticales e impositivas, que son las que están latentes en un régimen disciplinario, en el que hay un brazo ejecutor que generalmente no atiende a justificaciones individuales y lo único que hace es aplicar lo que el reglamento dice que hay que hacer en cada caso.

Pero a pesar de la "despersonalización" y la "standarización" de las relaciones que supone el diseño abstracto de un régimen disciplinario, no quiere decir que no debamos tener normas de actuación o un marco de referencia que "regularice" nuestras actuaciones. No estamos hablando del laissez faire, o de dejar de poner límites. Pero sí que debemos replantearlo, re-conducirlo hacia una re-definición de esas actuaciones que lleven a los reincidentes, a los de conductas disruptivas, a los carentes de "límites" en sus conductas, a elaborar lo que podríamos denominar como un programa personalizado de asumir responsabilidades sobre el daño hecho a la comunidad educativa o a personas miembros de ésta, para contraer unos compromisos para reparar o contrarrestar dichos daños.

Evidentemente, es aconsejable que las acciones sean coordinadas desde los distintos ámbitos de actuación, esto es: a nivel curricular, a nivel tutorial y de trabajo de aula y de centro, pero no siempre se logra tal coherencia. Por eso, cualquier paso puede ser el 
primero; lo importante es empezar a andar, no es necesario esperar a que se conjuguen todas las condiciones para iniciar la intervención, ya que supondría simplemente postergar lo que podemos empezar a hacer ya mismo, aunque en pequeñas medidas.

\section{Resolución de conflictos y habilidades sociales: cómo integrarlos a los programas de convivencia de aula y de centro}

Para la educación para la paz y para la resolución de conflictos es necesario el conocimiento del entorno sobre el que se realizará la intervención para vincular la idea de paz no utópica sino cotidiana, no de intervenciones de paz, sino en las rutinas domésticas, en las relaciones interpersonales. Pero somos conscientes de que la mera información, aún siendo imprescindible, es insuficiente para educar para la paz, para modificar o promover determinadas conductas.

Gracias a estos métodos de resolución de conflictos también propiciaremos modelos más activos de participación y más responsables, ya que ofrecen procedimientos de expresión pacífica de los conflictos, y formas de canalizarlos. Por lo tanto, es una oportunidad valiosa para la construcción de consenso y esto siempre es muy revitalizante en las instituciones, porque estaremos enseñando a participar en una sociedad democrática. Si adoptamos esta actitud, estaremos cambiando las posturas antagonistas por las protagonistas y, en consecuencia, estaremos educando para la paz.

En el marco del sistema educativo implica necesariamente la formación de criterios éticos que sean compatibles con determinados modelos sociales e ideológicos, dejando de lado otros. Esta situación abre una serie de interrogantes sobre varios dilemas a dilucidar, por ejemplo sobre los valores que se han de fomentar, en donde se incluirá y también a cuál visión de paz ha de darse prioridad. Si adoptamos en nuestra cotidianeidad un método de resolución cooperativo del conflicto -sin eludirlo, sino por el contrario haciéndole frente con las herramientas adecuadas-, donde se toman en cuenta las necesidades de todas las partes implicadas, estaremos ofreciendo implícitamente, en la práctica y las actuaciones de todos los días, cuál es el camino a seguir, estaremos haciendo una educación para la paz a través de enseñar la cultura de paz en nuestro entorno, con quienes nos rodean, en un continuo aprendizaje de la cultura de la paz por parte de todos, docentes, alumnos.

Para llevar a la práctica, en el día a día, la Educación para la Paz en el ámbito educativo, debemos tener en cuenta algunos aspectos de a) la organización de los centros, b) del tipo de actividades que se realizan y, en consecuencia, c) el tipo de relaciones que tienen lugar entre los distintos actores. Para acotar un poco más, las que aquí proponemos son herramientas para ayudar al profesorado en esos tres aspectos, para que desarrolle competencias en la resolución de conflictos tanto para sí mismo como para el asesoramiento o la actuación con otros. Para ello hemos seleccionado algunas cuestiones para la mejora en las habilidades sociales y de comunicación, el método PIGEP de autocontrol y toma de decisiones ante situaciones conflictivas, que a la vez todas ellas sirven para que las utilicemos para negociar con alguien con quien mantengamos una disputa, como así también para aplicar como mediadores, si tenemos que ayudar a dos partes enfrentadas a que lleguen a un acuerdo. 


\subsection{Aprender a expresar/comprender}

Este principio es básico, se remite a lo que entendemos por comunicación, pero pese a su simplicidad, pocas veces hacemos una auténtica "escucha activa" o sólo les enseñamos a los otros a hacerla. En lo referido al modelo de comunicación clásico, esto lo situamos en el polo de la recepción, y por ello nos referimos a la "comprensión". Comprender es escuchar, pero es más que eso, es entender, empatizar, interesarse por el otro y aún más, es también ponerse en su lugar.

En el otro polo de la comunicación está la emisión y a ella se refiere lo de "expresar", pero nos referimos a expresar ¿cómo?. No es cuestión de expresar sin más, de exteriorizar lo que queremos decir tal como nos venga, sino de expresarnos asertivamente, de un modo no acusatorio, utilizando los "mensajes en yo". Es decir, consiste en responder poniendo límites, y no permitir abusos o malos tratos como supone la respuesta "pasiva" o reaccionar violentamente como es en el caso de la respuesta "agresiva". Con ello estamos enseñando a que la paz, la armonía no se basan en consentir el abuso, en ser sumisos, en no defenderse, sino que estamos promoviendo habilidades para "hacer que nos respeten, respetando".

Estos son algunos consejos para empezar a "limpiar" la comunicación, sacarle sus elementos "contaminantes". Estas, que parecen dos herramientas muy sencillas, son muy complejas de poner en práctica, pero aun así, no está demás tener en cuenta que se acuerden, por ejemplo a nivel del trabajo de aula, unas normas de comunicación que permitan que todas las voces sean escuchadas (fijando turnos de palabra, promoviendo la participación de todos, etc.) y no permitiendo estilos de comunicación verbal o no verbal que denigren a las personas o deterioren las relaciones entre éstas (rumores, insultos, etc) o al entorno.

Una buena comunicación es imprescindible a la hora de resolver los conflictos escolares. Una comunicación interpersonal de calidad a nivel personal tenderá a producir:

a) Un descenso del temor a ser rechazado.

b) Una disminución de la ansiedad generada a partir de la lucha por la aceptación y el reconocimiento.

c) El incremento de la predisposición a escuchar al otro y a reconocerle sus aspectos positivos.

d) Un refuerzo de la autoestima, un aumento del grado de seguridad ontológica y una disminución, por ende, de las conductas defensivo ofensivas.

De esta manera, contar con canales de comunicación fluidos y de calidad proporcionará una serie de ventajas en el clima de convivencia:

* permite una organización cooperativa,

* ayuda a resolver problemas, y no queden enquistados,

* proporciona una mayor seguridad individual, contribuyendo a la autoestima y a la autovaloración, y también a la autonomía y la responsabilidad,

* aumenta la comunicación, ya que se manifiesta eficaz para atender a las cuestiones personales y grupales. 
Algunos de los mecanismos facilitadores de la comunicacióna a tener en cuenta son:

\section{a) La empatía}

Los obstáculos mencionados anteriormente nos hacen concluir que para crear empatía se debe respetar al otro, sus opiniones y puntos de vista, sus valores, decisiones y experiencias. La empatía es lo que hace que haya una buena comunicación, el nexo entre dos personas que permite que comprendan mutuamente sus sentimientos y motivaciones.

Para que la empatía funcione, no hay que mezclar la relación ni a las personas con el problema, hay que trabajarlos independientemente, por separado. También será necesario en el centro y en el aula que se cree grupo, es decir el sentimiento de pertenencia y cohesión será un elemento necesario para poder apelar al compromiso y a la responsabilidad, al efecto de nuestras acciones sobre los otros, promoviendo la interdependencia y la confianza.

\section{b) Feed-back}

Apoya y estimula modos de comportamiento positivos, corrigiendo los inadecuados, y ayuda a comprender mejor al otro. Para ponerlo en práctica debe hacérsele saber al otro lo que uno siente y piensa sobre sí mismo y sobre el otro, haciendo esto, a su vez, recíprocamente. Tiene en cuenta las necesidades de los implicados, está orientado al comportamiento que puede ser modificado. No se impone, por lo cual debe examinarse la buena disposición de los implicados, es decir, la voluntariedad y que no haya actitudes defensivas. También ha de considerarse que el momento sea adecuado, las motivaciones que lo impulsan así como la relevancia de la información que se trata para los implicados. Cuando ya se establece la comunicación, la empatía aumenta. Deben explicitarse así mismo los sentimientos, para que genere confianza y la consecuente retroalimentación, que es lo que garantiza que este tipo de relación se mantenga y, según evolucione, se consolide.

\section{C) Escucha activa}

¿Estamos realmente escuchando o solamente esperando que el otro acabe para empezar a hablar? Esta es la pregunta clave para determinar si realmente escucho o escucho activamente. Significa dejar de lado el propio punto de vista para "sintonizar" con el del interlocutor. Esta herramienta es bueno utilizarla para obtener más información, corroborando datos, para que el interlocutor sepa que ha sido escuchado con exactitud. Hace del diálogo un intercambio de información auténtica y no monólogos complementarios o superpuestos.

Cuando escuchamos activamente estamos preguntando, parafraseando, pidiendo aclaraciones, acotando y contextualizando. Algunas formas de utilizarla pueden ser por el eco (repetición de lo que ha dicho el otro), la reformulación (expresar con las propias palabras lo que se ha entendido), aclarando puntos o temas en cuestión, resumir y ordenar información o el reflejo del sentimiento, que es la expresión de lo que hemos percibido del otro. 
Las pautas a seguir son:

- No hablar de uno mismo.

- No aconsejar, diagnosticar, tranquilizar, animar, amenazar, sermonear, criticar u hostigar.

- No dar lecciones, mandar, consolar, aprobar o desaprobar, interpretar o ironizar.

- No ser sarcástico, no ridiculizar, descalificar o subestimar al otro o a sus sentimientos.

No es sólo devolver información o impresiones, es también escucha pasiva, es decir, comunicación analógica, el silencio interesado o expresiones gestuales (faciales, corporales, miradas, etc.) de aceptación y receptividad, mostrando interés; es comprender empáticamente, es decir, poniéndose en el lugar del otro.

\section{d) Asertividad}

Consiste en ser capaces de exponer el propio punto de vista, emociones u opiniones sin provocar una actitud defensiva. Es explicar cómo se ven las cosas, cómo es la situación desde la perspectiva personal en lugar de decir qué deberían o no hacer los demás. No significa retirarse, pero tampoco combatir. Es una frase autoafirmativa que le dice a la otra persona lo que se piensa sin culpar al otro, sin exigirle cambios, no se lo pone como contrincante. Es distinto responder a reaccionar. Es un instrumento para la consecución de un fin, a la vez de un medio para quedar satisfecho consigo mismo, ya que no permite la invasión del "territorio" personal, pero tampo invadimos el del otro.

Requiere comprender las propias limitaciones y las del otro, por lo que se favorece el autorrespeto y el de los demás. Establece su posición con claridad y genera autoconfianza. Permite a la otra persona saber que se le comprende y cómo uno se siente. Por lo que pone límite a situaciones de abuso, sin consentirlo ni agredir al otro, como hemos comentado más arriba.

\subsection{Aprender a disentir/consensuar}

\section{El cambio de actitud necesario}

Para enfocar correctamente la educación para la paz es necesario considerar la diversidad y la diferencia como un valor, como una oportunidad de crecimiento. Vivimos en una sociedad con multiplicidad de matices y diferencias, y en la pluralidad debe haber un espacio para la diversidad, la cooperación y la solidaridad, ya que ello representa una fuente de enriquecimiento. Pero esa diferencia, tradicionalmente se lo ha percibido como fuente de divergencias o disputas, en lugar de ser origen de crecimiento personal y grupal.

No estamos acostumbrados a que piensen o actúen distinto de nosotros. Llevamos muy mal que nos contradigan. Es muy baja nuestra tolerancia hacia lo distinto y esto mismo es parte de un aprendizaje que no está garantizado ni por los años que llevamos viviendo en democracia ni por la educación en valores. ESTAS CONMIGO O ESTAS CONTRA Mí es una actitud que a nivel cotidiano deteriora mucho las relaciones y hace imposible la CONVIVENCIA. 
Al toparnos con la intolerancia al disenso o a las diferentes formas de actuar y pensar debemos complementarlo con un trabajo de "consensuación", es decir, que podamos convivir con la diferencia, sin que ello signifique irreconciliable, traumático, que sea fuente de malestar. Que el disentir o rechazar una forma de pensar o actuar de una persona no implique el rechazo a la persona.

Para esto nos apoyaremos en la fórmula aportada por Elizabeth Crary, para saber cómo proceder ante un conflicto y que se puedan llegar a pequeños acuerdos que vayan limando las asperezas y, a la vez, los alumnos aprendan a NEGOCIAR. Una habilidad prosocial, que será de gran ayuda para el desarrollo de sus competencias emocionales o, simplemente, su mejor adaptación socio-afectiva.

Parte de las "diferencias" que mantenemos con otros son las que hacen de nuestro paso por los grupos, experiencias de crecimiento, aprendizaje y superación personal. En el aprender a congeniar, complementar y negociar nuestros distintos puntos de vista y elecciones. $Y$ en eso consiste el llevar a términos prácticos y concretos la convivencia, la educación para la paz en nuestro entorno, no en acallar el disenso, optando por la pasividad para evitar el enfrentamiento, sino en dar protagonismo y responsabilidades a los participantes, en crear y consensuar normas, en desarrollar las habilidades negociadoras para lograr y mantener los acuerdos, en asumir los consecuentes compromisos, en dividir roles y funciones democráticamente, creando mecanismos de participación, de toma de decisiones y de resolución de conflictos, y siempre esto desde la tolerancia y el respeto.

Alterar la convivencia puede alterar los compromisos, la tolerancia, el respeto de los otros o de sus derechos, y esto afecta a miembros o a valores del sistema, afecta al clima del grupo, provocando malestar en sus integrantes. La resolución pacífica y constructiva de conflictos se opone al deterioro que puede ocasionar el paso por grupos que no permiten la expresión y realización de sus miembros.

Motivar en la tarea y en el "compartir", es decir crear grupo, para que se creen vínculos de pertenencia, de complicidad y de confianza recíproca y cooperación son condiciones necesarias para facilitar la comunicación y la actitud positiva hacia las diferencias.

Lo que se busca por este método es hablar sobre el conflicto, sacándolo de la emocionalidad, llevandolo a la racionalidad, para de este modo poder tomar distancia e ir a la negociación siendo capaces de expresarnos, sacar las necesidades de las partes implicadas y buscar soluciones para satisfacerlas. Se intenta por medio de estos procesos de consenso que se permitan a las partes la resolución de conflictos a pequeña escala siendo ellos mismos los protagonistas, que sean capaces de asumir responsabilidades en la "reparación" del daño material o el daño hecho a la comunidad y a las personas que la integran, o en las consecuencias de su acción, pero que también se asuma el perdón y la reconciliación como actitudes necesarias para la tolerancia, el respeto y la convivencia.

\section{Distintas actuaciones ante el conflicto}

Comentábamos más arriba sobre cómo traducir el cambio de actitud ante el conflicto, ante discrepancias con acciones concretas. En primer lugar explicaremos una 
estrategia individual, que sirve también para aplicar en negociaciones(dos partes encontradas) y en mediaciones (dos partes encontradas y un tercero que de forma imparcial asiste a las dos partes en su negociación).

\section{ACCIÓN INDIVIDUAL:}

El método es sencillo pero eficaz y ayuda a aclarar el problema, a la toma de decisiones y a canalizar el conflicto hacia la reflexión; es una forma de autocontrol. Elizabeth Crary* lo denominó como PIGEP, que consiste en:

- Parar: cuando se detecta el malestar o enfado no reaccionar sino detenerse a pensar lo que se va a decir y a hacer. No dejarse llevar por el primer impulso.

- Identificar: definir el problema, diferenciar las necesidades de los deseos. Hablar con el otro sobre su versión de los hechos y contarle la propia. Evitar las etiquetas o juicios generales que obstaculicen el "poder hablar".

- Generar: ideas espontáneas, posibles soluciones, que sean distintas propuestas para resolver el problema, sin restricciones. La racionalidad de las mismas se sopesará en el paso siguiente.

- Evaluar: entre todas las ideas seleccionar las más viables, las que más satisfagan a las necesidades de cada una de las partes.

- Planificar: la puesta en marcha de las soluciones propuestas previendo los posibles obstáculos que puedan surgir para hacerla lo más realista posible. Elaborar un plan, agenda o contrato para implementarlo.

Desde luego, la armonía personal, la adquisición de ciertas actitudes que favorezcan la comprensión y el diálogo y la tolerancia son requisitos indispensables, pero insuficientes por sí solos. La forma de intervenir en los conflictos es esencial en la educación para la paz, no sólo en cuanto a los contenidos, sino también como una serie de procedimientos vitales en las relaciones interpersonales, en el marco de los grupos e intergrupales. De allí que aparezcan como muy necesarios prestar importancia a aspectos tales como cooperar, decidir de forma responsable, aprender a resolver, negociar y regular los conflictos. En el docente está la misión de promover en su comunidad educativa unas reglas de juego comunes a todos sus miembros, para garantizar esta igualdad en la participación. Cada uno de estos aspectos son algunas de las piezas con las que construímos y completamos el puzzle de la "educación para la paz" y la concretamos en el trabajo diario.

\section{LA NEGOCIACIÓN:}

Aquí deberemos aplicar esas habilidades que hemos aprendido a nivel individual para conciliar las diferencias que exista con otros. Así deberemos diferenciar distintos tipos de negociación:

* En Elizabeth Crary: Crecer sin peleas. Cómo enseñar a los niños a resolver conflictos con inteligencia emocional. Ed. Integral, Barcelona, 1994, p. 192. 
a) Negociación competitiva : existe un alto interés por uno, bajo por el otro. En función de la conducción del conflicto: las dos partes quieren imponerse, lleva a la mutua destrucción. Lo que busca es llevar al conflicto a que uno gane y el otro pierda.

b) Pacto o capitulación: en el que existe un alto interés por lo que se negocia, por lo que hay en juego, y bajo interés por una o ambas partes implicadas o por el vínculo. Una o ambas partes ceden algo. Las relaciones son destructivas: por una parte conduce a un renunciamiento o retirada y, por la otra parte hay dominación (una parte se intenta imponer a la otra). Conduce la disputa en términos de que ambas partes ganan y pierden un poco. Es la línea de negociación próxima al regateo.

c) Negociación cooperativa: en ésta existe un alto interés por el otro (sin renunciar a lo propio). Conducción constructiva del conflicto: supervivencia de las relaciones. Lleva a acuerdos del tipo ganar-ganar. Es en esta línea de negociación que debemos trabajar. Debe concebirse siendo duros con el problema y suaves con la persona, separando a uno del otro. Este modelo de negociación es el desarroIlado por Fisher y Ury y para llevarla a cabo nos recomiendan tener en cuenta siete elementos: los aspectos de la comunicación y de la relación que ya hemos mencionado, más la actitud ante los compromisos así como el marco de la legitimidad en el que se arriba a acuerdos. Teniendo en cuenta estas premisas, este modelo hace hincapié en diferenciar las posiciones de los intereses, que los podemos distinguir porque las posiciones responden a la pregunta ¿qué quieren? y los intereses los descubrimos en el ¿para qué lo quieren?. Así mismo no estará demas analizar cuáles son los intereses subyacentes, para luego crear opciones y alternativas. Las primeras orientan las posibilidades de máxima: qué es lo mejor que me podría pasar y las segundas las de mínima: ¿qué es lo peor que me podría pasar?¿ cuáles serán las consecuencias si no llegamos a un acuerdo?

Consideran estos autores que con este modelo de negociación se conduce a acuerdos más equilibrados y mutuamente satisfactorios.

\section{LA MEDIACIÓN: LOS PEACEMAKERS}

La que nos queda por comentar, en último lugar es la mediación escolar. Es última no por menos importante, sino por más compleja, ya que necesitaremos de todas las habilidades previamente comentadas para poder intervenir como mediadores. Este es el modelo conocido como de peacemakers o pacificadores. Su surgimiento estuvo estrechamente vinculado a los movimientos de paz, pero no se llama así sólo por eso. En el orden internacional, es común oír hablar en los movimientos diplomáticos y en la intervenciones de paz de los mediadores. Llevar a los centros esta figura que practicaría la diplomacia a pequeña escala y en conflictos cotidianos.

2. La negociación colaborativa está explicada en las siguientes obras:

- FISHER, R. y ERTEL, D., Sí... ¡De acuerdo! En la práctica, Bogotá, Colombia, Ed. Norma, 1998.

- FISHER, R.; URY, W. y PATTON, B., Obtenga el sí. El arte de negociar sin ceder, Barcelona, Ediciones Gestión 2000, S.A., 1996. 
En algunos programas de mediación escolar, los mediadores son los propios alumnos, que realizan mediación entre sus propios compañeros, que es el modelo más extendido. Es la conocida como mediación entre iguales. También está el modelo de mediadores adultos (interno o externo al centro), es decir un mediador profesional que es contratado por un centro para resolver un conflicto o un profesor o un padre que conoce el método y trata de colaborar cuando surge un problema. La ventaja del primer método con respecto al segundo es que el primero fomenta la autonomía en la resolución de conflictos entre alumnos, además del modelado que supone el ver a un compañero que posee las habilidades para ayudar a resolver un conflicto que los propios protagonistas no son capaces, por lo que produce a la vez un efecto multiplicador; promueve la armonía el el grupo de pares, y no por imposición de los adultos.

En el segundo modelo los alumnos siguen dependiendo de la intervención de los adultos para poder resolver, aunque con la ventaja de que no es con una solución impuesta. El primer modelo de mediación, la de entre iguales, tiene un inconveniente, que es el tiempo que hay que invertir en la formación y entrenamiento de los mediadores, pero es la única manera para imbricarla en la cultura del centro.

El último modelo que comentaremos es el de la comunidad de Madrid, ya que no tenemos conocimiento de otras experiencias similares, que es el de mediación en toda la comunidad educativa. En él, profesores, alumnos, padres, agentes externos, no docentes, etc. forman parte del proyecto. Todos realizan el entrenamiento y entre todos conforman el "servicio de mediación del centro". Ante lo cual la compenetración de la política educativa, de la gestión de conflictos, de la convivencia y en definitiva, de educación para la paz, es mucho mayor.

El programa de mediación escolar se basa en la negociación colaborativa. En ella una tercera persona, el mediador, que puede ser un alumno del centro formado para esa función, ayuda a las partes en conflicto a que encuentren una solución, facilitando la comunicación para la búsqueda del bien común, creando por esta vía un mecanismo autónomo de resolución de conflictos en el centro, ya que por este método las partes implicadas son las que elaboran las posibles soluciones conjuntamente, evitando de esta forma que otros resuelvan por ellos y que se apliquen soluciones impuestas. El mediador sólo regula el proceso.

Un objetivo de este tipo de mediación es que los alumnos acaben por llevar a sus hogares las habilidades de mediación aprendidas en el ámbito escolar, reafirmándose como un modo de relación general tendente a la paz, aquí también una vez más el efecto multiplicador es fundamental, porque lo que empieza siendo clima de centro llega a ser parte del clima de una comunidad educativa toda.

En estos programas se les explica a los alumnos el significado de conceptos como "comunicación", "conflicto" y "negociación". Luego, los alumnos que quieren seguir adelante evalúan sus propia capacidades para el desempeño como mediadores y si deciden que se sienten capaces de hacerlo, comienzan a mediar cuando aparecen los conflictos.

En la escuela es un proceso voluntario, absolutamente confidencial y con normas muy claras que tienden a crear un clima de cooperación. Cuando se produce un problema entre dos o más alumnos, una de las partes, si así lo quiere, puede recurrir a 
los alumnos mediadores -que en general actúan por parejas-. Si la otra parte lo acepta se lleva a cabo una reunión donde cada uno da su versión del problema.

Los mediadores no buscan culpables porque no son jueces. Tampoco dan la solución al problema sino que ayudan a buscarla. Si el conflicto se resuelve favorablemente, las partes pueden firmar un acuerdo por escrito o hacerlo verbalmente. Pero no están obligados a llegar a un acuerdo si no están satisfechos con las potenciales soluciones.

Estos programas recogen como precedentes las experiencias de finales de los años 60 , principios de los 70, de los religiosos y los pacifistas que buscaban formas alternativas de resolver los problemas de violencia que repercutían en el sistema educativo en la convulsionada sociedad americana que en esos años sufría la guerra, movimientos sociales, étnicos, pacifistas, etc.

Los objetivos de estos programas son no sólo prevenir la violencia y las peleas entre alumnos, son también enseñar mejores estrategias para la solución de problemas y la toma de decisiones, mejora la comunicación por lo tanto también las interrelaciones personales, favoreciendo esto al clima de aula y de centro. Los beneficios en las relaciones contribuyen a una disminución de los problemas disciplinarios, porque el diálogo se impone donde antes predominaba el castigo, las imposiciones, la falta de respeto y los insultos y las agresiones.

Quiere decir que, a nivel institucional crea canales para la resolución de conflictos, de búsqueda de soluciones constructivas, no destructivas, que contemplen el beneficio mutuo, cuya gestión, además de solidaria, es autónoma y participativa, contribuyendo, consecuentemente, al fomento de vínculos más democráticos en el centro, atendiendo a la vez tanto a la diversidad como al desarrollo del sentido de comunidad, creando un mayor sentimiento de compromiso, tanto en la dimensión personal como en la comunitaria.

\section{3. ¿Y cómo se relaciona todo esto con la Educación para la Paz?}

Educar para la paz no es sólo educar contra la guerra. La Paz no es algo inasible, de dirigentes y foros internacionales o de simbólicas e idílicas palomitas, sino que es una paz en el entorno cotidiano, de resolución de conflictos a pequeña escala, es decir de paz activa, de soluciones y aportaciones constructivas, de protagonismo de los participantes.

Lo que está claro es que la cultura de paz se opone a la disciplina en su acepción más autoritaria. Esto se produce como resultado de otra de las consecuencias que ha generado la experiencia de mediación en la comunidad de Madrid: el cuestionamiento al modelo punitivo. Esta experiencia promovió en los centros una auténtica y revitalizante reflexión hacia la "cultura del castigo" predominante, que condujo a un replanteamiento del tradicional sistema de sanciones para el mantenimiento de la disciplina. Llevó a repensar los límites de la disciplina y en consecuencia los de la autoridad, que son los gestores de la aplicación de un determinado regimen y modelo de convivencia., son los que dicen lo que está bien y lo que está mal y cuándo y cómo se debe castigar. Alterar la convivencia puede alterar la tolerancia, el respeto de los otros o de sus derechos y deberes, el ejercicio de las responsabilidades y obligaciones 
de sus componentes y esto afecta a valores del sistema y al clima del grupo, provocando malestar en sus integrantes. El deterioro que puede ocasionar el formar parte de grupos que no permiten la expresión y realización de sus miembros se puede revertir creando una cultura de paz.

No hablamos de paz de los cementerios, porque nada se mueve, hablamos de paz activa; la no confrontación que algunos han entendidos como un condición intrínseca a la paz, supone la pasividad, el consentimiento, la no agresividad. Hemos expresado que habilidades y actitudes necesitaremos poner en práctica para que la paz salga de los expertos de los foros internacionales y de las ONGs y entre en nuestra vida cotidiana, a nivel interpersonal, de aula, de centro y comunitario.

Con estas políticas de gestión de conflictos, hacemos de las partes integrantes auténticos protagonistas, que participan y asumen compromisos y por lo tanto habrá disenso, porque no somos todos iguales y porque en eso, precisamente en eso, reside la riqueza de los grupos (en la variedad está el gusto). Pero parte de esas "diferencias" son las que hacen de nuestro paso por los grupos experiencias de crecimiento, aprendizaje y superación personal. En el aprender a congeniar, complementar y negociar nuestros distintos puntos de vista y elecciones.

En eso consiste el llevar a términos prácticos y concretos la cultura de paz, no en acallar el disenso sino en dar protagonismo y responsabilidades a los participantes, en crear y consensuar normas de convivencia, en desarrollar las habilidades negociadoras para lograr y mantener los acuerdos, en asumir los consecuentes compromisos, en dividir roles y funciones democráticamente, creando mecanismos de participación, de toma de decisiones y de resolución de conflictos, y de tolerancia y de respeto.

Incorporando estas habilidades en la enseñanza podremos hacer de la educación un medio eficaz para socializar, no para la mera transmisión de conocimientos sino para facilitar la integración en la sociedad y para promover las conductas socialmente deseadas y valoradas e intentar, de esta manera, hacer de nuestro paso por los grupos un aprendizaje de convivencia.

\section{Referencias bibliográficas}

ALZATE SÁEZ DE HEREDIA, R. 1998 (octubre). El papel de la mediación dentro de los programas de resolución de conflictos en el ámbito escolar. Mollet del Vallés, II Jornades Catalanes de Resolució Alternativa de Conflictes.

APDH. 1996. Seminario de Educación para la Paz. Educar en y para los derechos humanos. Madrid, Los libros de la Catarata.

CARBONELL FERNÁNDEZ., J. L. (coord.). 1997. Convivir es vivir. Programa de desarrollo de la convivencia en centros educativos de la Comunidad de Madrid. Madrid, Ed. Obra Social de Caja Madrid.

CASAMAYOR, G. (coord.). 1998. Cómo dar respuesta a los conflictos. La disciplina en la enseñanza secundaria. Barcelona, Ed. Grao.

CASCÓN, P. y BERISTAIN, C. M. 1999. La alternativa del juego. Juegos y dinámicas en la educación para la paz. Madrid, Los libros de la Catarata (4⿳亠丷a ed.). 
CASCÓN, P. 2000 (enero). "Educar en y para el conflicto en los centros", en Cuadernos de Pedagogía, no 287. Barcelona, pp. 61-66.

COLECTIVO AMANI. 1994. Educación intercultural. Análisis y resolución de conflictos. Madrid, Ed. Popular.

CORNELIUS, H. y FAIRE, S. Tú ganas y yo gano. Cómo resolver conflictos creativamente y disfrutar con las soluciones. Madrid, Gaia Ediciones.

CRARY, E. 1994. Crecer sin peleas. Cómo enseñar a los niños a resolver conflictos con inteligencia emocional. Barcelona, Ed. Integral.

FERNÁNDEZ ENGUITA, M. 1995. La escuela a examen. Un análisis sociológico para educadores y otras personas interesadas. Madrid, Ed. Pirámide.

FERNÁNDEZ GARCÍA, I. 1998. Prevención de la violencia y resolución de conflictos. El clima escolar como factor de calidad. Madrid, Narcea.

FISHER, R. y ERTEL, D. 1998. Sí... ¡De acuerdo! En la práctica. Bogotá (Colombia), Ed. Norma.

FISHER, R.; URY, W. y PATTON, B. 1996. Obtenga el sí. El arte de negociar sin ceder. Barcelona, Ediciones Gestión 2000, S. A.

GERNIKA GOGORATUS. 1998. Caja de herramientas del I curso internacional de capacitación para el entrenamiento en tratamiento de conflictos. Gernika, ed. Gernika Gogoratus (14 al 20-9-98).

GIRARD, K. Y KOCH, S., Resolución de conflictos en las escuelas. Manual para educadores, Bs.As., Ed. Granica, 1997.

GOTTHEIL, J. y SCHIFFRIN, A. 1996. Mediación: una transformación en la cultura. Buenos Aires, Paidós Mediación.

GRASA, R. 2000 (enero). "Evolución de la educación para la paz", en Cuadernos de Pedagogía, no 287. Barcelona, pp. 52-56.

JARES, X. 1991. Educación para la paz. Su teoría y su práctica. Madrid, Ed. Popular.

LEDERACH, J. 1985. La regulación del conflicto social: un enfoque práctico. Akron (USA), Ed. Comité Central Menonita.

PANIEGO, J. A. 1999. Cómo educar en valores. Métodos y técnicas para desarrollar actitudes y conductas solidarias. Madrid, Editorial CCS.

ROZENBLUM DE HOROWITZ, S. 1998. Mediación en la escuela. Resolución de conflictos en el ámbito educativo adolescente. Buenos Aires, Aique. 